\title{
Recognition and Analysis of Human Facial Expression: A PSO Optimization Method
}

\author{
Zhijie Li, Xiaodong Duan ${ }^{+}$, Cunrui Wang \\ ${ }^{1}$ School of Computer Science \& Engineering, Dalian Nationalities University, Dalian Liaoning \\ 116600, China \\ ${ }^{2}$ Dalian Key Lab of Digital Technology for National Culture, Dalian Nationalities University, Dalian \\ Liaoning 116600, China \\ + Corresponding author email: minzudigital@dlnu.edu.cn
}

Keywords: Facial expression recognition, Face detection, Particle swarm optimization, Principal component analysis

\begin{abstract}
A method for precise face detection and segmentation combining with the particle swarm optimization is proposed. The method uses three key technologies: skin color segmentation, particle swarm search and curve approximation. The experimental results show that this method can eliminate the interference factor, and improve the accuracy of expression recognition, especially well for some single expression recognition.
\end{abstract}

\section{Introduction}

Research on expression recognition has a development history about thirty years, since the earliest facial expression recognition system is established by Suwa and Sugie [1] in 1978. It has been found that the existing studies on expression recognition mainly contain two categories: (1)one is expression recognition focus on different person. The typical methods contains: Andrew and Calder[2] made a detailed introduction of the PCA in facial expression recognition. Chen Xuewen[3] combined cluster method with linear discriminate analysis (LDA) on the facial expression feature extraction, and obtained a high recognition rate on single expression. (2)The other is expression recognition focus on the same person, namely dependent of specific person. This kind of recognition matches the input image with all expressions of the same person in the expression database. Then, sort the matching data to output the expression classification information as well. Its significant characteristic is that all the face images are collected from the same individual. Expression recognition focus on the same person is usually used to investigate individual emotion change. Relevant studies are rare. The typical research is in 2011, Tanveer[4] developed a face expression classification system based on PCA. But the recognition accuracy of the system needs to be improved further.

This paper focuses on the second category of expression recognition problem-recognition for the same individual. Expression recognition requires the image containing exaggerated and obvious expression. How to effectively improve the expression recognition accuracy? We think that as the important step of expression recognition, face feature extraction determines the accuracy of expression recognition. While current research on feature extraction owns many mature methods, but preprocess such as face detection is not paid enough attention. This is the reason for the limitation of recognition accuracy. We combine the particle swarm optimization algorithm (PSO) [5] and the skin color segmentation to deal with the face detection and segmentation, and thereby improving the following facial expression recognition.

\section{Solution for Improving Recognition Accuracy}

Particle swarm algorithm is a global optimization algorithm; it can solve a large number of non-linear, non-differentiable and multi-peak complex optimization problems. Individual in PSO represents a possible solution. It is known that, most information is stored on the image edge. Also, face location is based on the edge information. After image preprocessing, a binary image of face 
image can be obtained. The next work is all performed on the edge of binary image. Face region can be located precisely using particle swarm searching on the edge image, which will improve the accuracy of expression recognition.

We have the following assumptions. The face position detection is proceeded under the assumption that the face region can be surrounded by a rectangle. This method works well even under the environments when the people pose is complex. It is commonly assumed in literature that the ratio of the length to width of the rectangle area is 1.5: 1, once the face region is located. But there is no limit on the size of rectangle since we don't know the scale of face in the image. The rectangle has three parameters: the abscissa $(x)$ and ordinate $(y)$ of the upper left corner, the length of the diagonal (r). The criterion of feature extraction is defined by the number of edge pixels in the rectangle area to the rectangle perimeter. The ratio is large when the rectangle contains edge points at more frequent. The criterion is calculated below.

$$
\begin{aligned}
\text { criterion } & =\frac{1}{2\left(x^{\prime}-x+y^{\prime}-y\right)} \sum_{i=x}^{x^{\prime}} \sum_{j=y}^{y^{\prime}} e(i, j) \\
\text { where } & \begin{cases}e(i, j)=1 & \text { if pixel is black } \\
e(i, j)=0 & \text { if pixel is white }\end{cases}
\end{aligned}
$$

$e$ is edge image of the original image and $e(i, j)$ represents a pixel point with value 1 or $0 .(x, y)$ denotes the coordinate of the upper left corner of the rectangle, while $\left(x^{\prime}, y^{\prime}\right)$ denotes the lower right corner of the rectangle. Thus, according to the ration of length and width, the following relationship exists.

$$
\begin{aligned}
& x^{\prime}=x+3 r / \sqrt{13} \\
& y^{\prime}=y+2 r / \sqrt{13}
\end{aligned}
$$

Based on the above analysis, as long as the largest ratio of rectangle is found, the task of face detection is completed. Next, we will utilize PSO algorithm to search the optimal rectangle region. Because of the length of this paper, we cannot detail the face detection using PSO here. The specific details can be seen in literature [6].

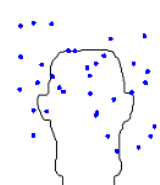

(a)

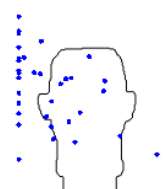

(b)

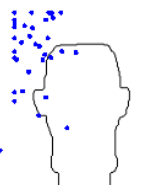

(c)

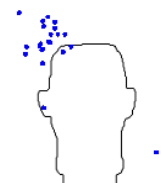

(d)

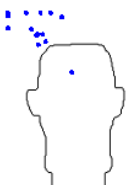

(e)

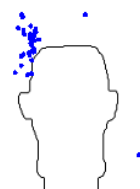

(f)

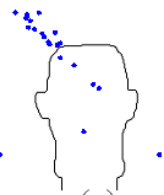

$(\mathrm{g})$

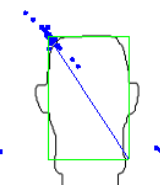

(h)

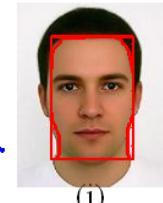

(1)

Fig. 1 Illustration of PSO searching process. (a)initial particle attribution; (b) 3 iteration; (c) 6 iteration; (d) 10 iteration; (e) 20 iteration; (f) 30 iteration; (g) 40 iteration; (h) 100 iteration; (i) final searching result

Fig. 1 intuitively illustrates the particle searching process on the edge image. The initial particles are randomly distributed on the face edge image. After several iterations, the particles convergent toward the optimal position. Obviously, in the former iterations, the particles fly quickly. After 10 iterations, particles gathered together. In the latter iterations, the particles fly slowly and adjust speed according to equation (1). After about 100 iterations, some particles find the optimal position, which is the coordinates and diagonal length of the upper left corner of the optimal rectangular. So, the face region is determined. The last figure (i) means face edge and rectangular searched by particles eventually are superposed together. The rectangular in figure (i) locates the face region precisely, excluding from hair, ear and neck.

\section{Expression Classification and Recognition}

After the above image preprocessing, we begin to extract the expression feature. There are many methods of simplify the data set or reduce dimension. Now, the widely used method is the principal component analysis (PCA). To simplify the studied problem, we labeled the face images using four expressions in the training set. Therefore, expression recognition problem can be regarded as distance matching problem among images, after feature extraction step. Matching is done through 
calculating the distance between the test images and the training images, according to certain distance criterion. Finally, based on the obtained similarity with various expressions, the facial expression is classified. Because there are not many feature points after dimension reduction, this paper takes the general Euclidean distance to define the true distance between any two points in multi-dimensional space. The shorter the distance, the more similar between two expressions.

Based on the above ideas, this paper uses particle swarm algorithm to carry on the simulation experiment for face segmentation and expression recognition, and compared with the results of the original algorithm for facial expression recognition results. Follows are the description of our facial expression database. The usually used expression database in expression recognition field is mainly focus on the recognition of different individual. However, the images for the same person in the same kind of expression are not many. Such as the Japanese female facial expression database (JAFFE) has 3 images for each person of each kind of expression on average. This number cannot meet our experiment requirement. Considering the above facts, we use the expression database provided by MI Tanveer. Each image size is $600 \times 800$. The training set contains 50 facial expression images, presenting five kinds of expression of the same face. Among them, neutral has 7 images, happy has 13 images, anger has 10 images, sad has 9 images and disgust has 11 images. These expressions are also labeled in a file. The test set contains 31 facial expression images. Among them, neutral has 4 images, happy has 8 images, anger has 7 images, sad has 2 images and disgust has 10 images. The test set select different expression images from those in training set, in order to measure the recognition ability after training.

The expression image is preprocessed according to the method in the above section. Steps include face detection, segmentation, and uniform. The preprocessing doesn't change the posture and expression of face image. In order to assess the recognition, we label and classify the expression images in the training set and test set. The preprocessed expression image size is uniformed as $180 \times 280$ bits. If the image is in the original space, its representation needs a vector of $180 \times 280$. But the image representation only needs a vector of $1 \times \mathrm{r}$ when using PCA technology to project it into some feature space. How to determine the $r$ value? As we know that the variance can be used to judge the importance of feature vector. For a random variable, the larger the variance, the more information contained. When the variance is zero, the variable is constant containing no information. PCA used to calculate the principal component, is to find a set of vectors making the variance of projection value from original data as large as possible. The vector corresponding to maximum variance is the first principal component. In the same way, we can get the second and third principal components. Through the calculation of 50 facial expressions in expression library, the eigenvector distribution corresponding to principal components is shown in Fig.2. From this figure, we can see that the importance of eigenvector decreased exponentially. Thus, only the front few important eigenvectors is needed to construct the Eigen space. On the observation of the Pareto diagram, we find the sum of the front 30 eigenvalues account for the $95 \%$ of the sum of all eigenvalues. That is to say, the contribution rate of the front 30 eigenvalues account for the $95 \%$ of the sum of contribution rate of all eigenvalues. So, the following principal components can be ignored. The $r$ value can be 30 instead of $180 \times 280$. The reduced $r$ value achieves the effect of reducing dimension. The following work is to select the front 30 principal components as the new subspace, and project all the expression images into this new subspace.

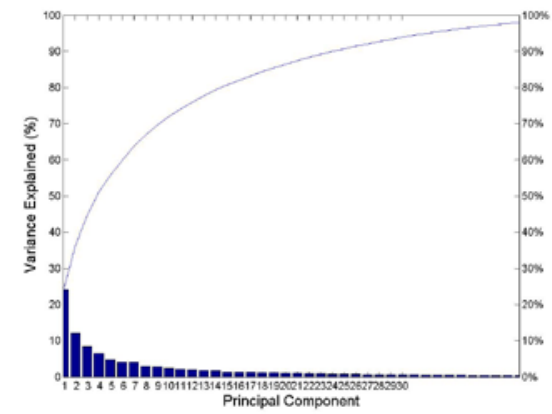

Fig. 2 Pareto diagram of contribution rate of forty principle components (eigenvalues) 
The Euclidean distances between the testing image and all the training images obtained by the Tanveer algorithm are compared with that obtained by the PSO algorithm. Obviously, the smaller the Euclidean distance of the two images, the more similar the images are. Therefore, we calculate the Euclidean distances of the test expression and all the training expressions, then select the minimum as the matching result. Two algorithms produce quite different results, but the PSO algorithm is correct. As to why the experiment can produce so big difference, we think it is mainly because that the preprocessed face contains too many interference factors in the Tanveer algorithm, which results in the inaccurate recognition. We verify the effectiveness of PSO algorithm through this typical example of expression recognition. Although the basic ideas of the two algorithms are the same, but the precise face segmentation of PSO algorithm has greatly improved the final recognition accuracy. This result shows the details determine success or failure. That is to say, the usually neglected face preprocessing plays a decisive role.

\section{Conclusion}

This paper investigates how to improve the accuracy of facial expression recognition, mainly focuses on the facial expression classification for the same individual. We refine the results of face preprocessing. The PSO algorithm is introduced into face detection and segmentation. Based on the skin segmentation, the PSO algorithm takes particles advantage of global optimization searching to face region, which removes the hair, ears, neck and other interference regions. The treatment plays the key role in expression recognition. Especially for the similar expressions, only the accurate face can get the correct matching results. After the expression image is preprocessed, the principal component analysis is used for face feature extraction and recognition. The experimental results on the special expression database show that facial expression recognition using PSO algorithm improves the recognition accuracy.

\section{Acknowledgement}

This work is supported by the National Natural Science Foundation of China under Grant No. 61370146, No. 61040054, and the Fundamental Research Funds for the Central Universities under Grant (No.DC201502030203, No.DC201502030403).

\section{References}

[1] M. Suwa, N. Sugie, K. Fujimora. A preliminary note on pattern recognition of human emotional expression, Proceedings of the International Joint Conference on Pattern Recognition, 1978, pp: 408-410.

[2] Andrew J.Calder, A Mike Burton, Paul Miller, et al. A Principal Component Analysis of Facial Expressions. Vision research, 2001, 41(9): 1179-1208.

[3] Chen Xuewen, Thomas Huang. Facial expression recognition: A clustering-based approach. Pattern Recognition Letters 2003, 24(9-10): 1295-1302.

[4] Md. Iftekhar Tanveer. Eigenface based Facial Expression Classification. http://www.mathworks.com/matlabcentral/fileexchange/33325-eigenface-based-facial-expression-cl assification. 2011, 11.

[5] Jian Zhang. Experimental Parameter Investigations on Particle Swarm Optimization Acceleration Coefficients. International Journal of Advancements in Computing Technology, 2012, 4(5): 99-105.

[6] Li Zhi-jie, Duan Xiao-dong, Liu Xiang-dong, Wang Cun-rui. Face Segmentation Based on PSO Algorithm. Advances in information science and service sciences. 2013, 5(3): 205-221. 